trum. If this shift were only 0.2 or $0.1 \mathrm{eV}$ however, significant amounts of this species could conceivably be obscured. In addition, spectra of the F $2 s$ level (shown elsewhere) ${ }^{5}$ revealed only a single approximately Gaussian shaped peak with a full width at half-maximum of $2.7 \mathrm{eV}$. Splittings in this level, which must occur in the polyfluoro groups, are therefore small. Seel and Bagus ${ }^{9}$ predict the F $1 s$ level of interstitial or threefold surface sites will exhibit large shifts from the polyfluoro $\mathrm{F} 1 s$ binding energy. While the $\mathrm{F} 1 s$ level is energetically inaccessible in our experiments, the photoemission behavior of the F $2 s$ is somewhat corelike; thus its single peak nature provides additional argument against the existence of interstitial fluorine for the conditions considered here.

In conclusion, we have identified the types of fluorosilyl moieties formed by the initial attack of fluorine atoms on clean silicon surfaces. The chemical shifts of approximately one, two, and three $\mathrm{eV}$ are characteristic of mono- $\mathrm{di}$-, and trifluorosilyl groups, independent of the details of surface structure. No evidence for interstitial or otherwise multiply coordinated fluorine atoms was found.

Finally, we note that difluorosilyl accounted for only a small portion of the total amount of fluorinated silicon found on either of the surfaces studied. Interestingly, a number of the kinetic models for the surface reaction involve difluorosilyl units as the most prominent surface species, presumably because $\mathrm{SiF}_{2}$ is a reaction product. If the same reaction mechanisms responsible for the initial stages of fluorine at- tack also dominate during the steady state reaction, then our results indicate these models are incomplete and that more emphasis should be placed on the reaction of trifluorosilyl groups.

We wish to acknowledge the staff of the National Synchrotron Light Source for providing the photons for these experiments.

'C. J. Mogab, A. C. Adams, and D. L. Flamm, J. Appl. Phys. 49, 3796 (1978).

${ }^{2}$ J. W. Coburn and M. Chen, J. Appl. Phys. 51, 3134 (1980).

${ }^{3}$ V. M. Donnelly and D. L. Flamm, J. Appl. Phys. 51, 5273 (1980).

${ }^{4}$ D. E. Eastman, J. J. Donelon, N. C. Hien, and F. J. Himpsel, Nucl. Instrum. Methods 172, 327 (1980).

${ }^{5}$ F. R. McFeely, J. F. Morar, N. D. Shinn, G. Landgren, and F. J. Himpsel, Phys. Rev, B (in press).

${ }^{6}$ H. F. Winters and F. A. Houle, J. Appl. Phys. 54, 1218 (1983); Yung-Yi Tu, T. J. Chuang, and H. F. Winters, Phys. Rev. B 23, 823 (1981).

${ }^{7}$ F. J. Himpsel, P. Heimann, T. -C. Chiang, and D. E. Eastman, Phys. Rev. Lett. 45, 1112 (1980).

${ }^{8}$ A. A. Baake, H. W. Chen, and W. L. Jolly, J. Electron. Spectrosc. Relat. Phenom. 20, 333 (1980).

${ }^{9}$ M. Seel and P. S. Bagus, Phys. Rev. B 28, 778 (1983).

${ }^{10}$ K. J. Gruntz, K. Ley, and R. L. Johnson, Phys. Rev. B. 24, 2069 (1981).

"The intensity of chemically shifted core levels $I_{s}$ relative to the total corelevel emission $I$, (shifted + unshifted) has been coverted into a coverage $\Theta$ (in monolayers) of fluorinated $\mathrm{Si}$ aotms using a continuum model:

$$
\Theta=(l / d) \ln \left[I_{t} /\left(I_{t}-I_{s}\right)\right]
$$

mean free path $l=4 \AA$, average layer spacing $d=1.565 \AA$.

${ }^{12}$ C. M. Garner, I. Lindau, C. Y. Su, P. Pinanetta, and W. E. Spicer, Phys. Rev. B 19, 3944 (1979).

\title{
Substitutional nitrogen impurities in pulsed-laser annealed silicon
}

\author{
Kouichi Murakami, Hisayoshi Itoh, Koki Takita, and Kohzoh Masuda \\ Institute of Materials Science, University of Tsukuba,Sakura, Ibaraki 305, Japan
}

(Received 30 August 1983; accepted for publication 4 May 1984)

\begin{abstract}
Single-crystal Si samples with nitrogen $(\mathbf{N})$ impurities $(\mathrm{Si}: \mathrm{N})$ and with $\mathbf{N}$ and phosphorus $(\mathrm{P})$ impurities (Si:N:P) have been investigated by electron spin resonance measurements. It was found that substitutional $\mathrm{N}$ impurities $N_{s}$ cannot be incorporated into $\mathrm{Si}$ by $\mathrm{cw}$ laser annealing of $\mathrm{N}$ ionimplanted $\mathrm{Si}$ or by $\mathrm{N}$ doping during crystal growth; however, $N_{s}$ is incorporated into $\mathrm{Si}$ by pulsedlaser annealing (PLA) of $\mathrm{N}$ ion-implanted $\mathrm{Si}$. The spin density of $N_{s}$ decreases with doping of $\mathrm{P}$ shallow donors into PLA Si:N and increases by introduction of slight point defects in PLA Si:N:P. These results suggest that $N_{s}$ with a negative charge are formed in PLA Si: N:P system.
\end{abstract}

It was clarified recently by electron spin resonance (ESR) measurements ${ }^{1-3}$ that substitutional nitrogen impurities $N_{s}$ can be introduced into silicon ( $\mathrm{Si}$ ) crystal by $\mathrm{N}_{2}^{+}$or $\mathrm{N}^{+}$ion implantation and subsequent pulsed laser annealing (PLA). At present, it is very important to explore techniques by which $N_{s}$ can be introduced into $\mathrm{Si}$, to investigate how deep the localized level of $N_{s}$ is in the neutral charge state, $N_{s}^{(0)}, 2$ and to determine whether negative charge states of $N_{s}$ exist in the band gap. ${ }^{4}$ In this letter, we report on ESR results of various kinds of $\mathrm{Si}$ samples including $\mathrm{N}$ impurities ( $\mathrm{Si}: \mathrm{N}$ systems) and on phosphorus (P) doping effects on Si:N systems.
All the ESR measurements were made at $9 \mathrm{Ghz}$ at room temperature (RT). Microwave powers lower than $2 \mathrm{~mW}$ were used to avoid the effects of absorption saturation. An ESR standard sample, $\mathrm{Mn}^{++}$in $\mathrm{MgO}$ with resonant magnetic fields different from those of $N_{s}^{(0)}$, was utilized to measure the spin density of $N_{s}^{(0)}$. Consequently, our evaluation of the spin density of $N_{s}^{(0)}$ was not affected by degradation in cavity $Q$ due to heavy $P$ doping, even though the ESR intensity decreased with increments in $\mathbf{P}$ concentration.

First, we made ESR measurements on three types of $\mathrm{Si}$ samples: PLA Si:N, cw laser annealed Si:N, and bulk $\mathrm{Si}: \mathrm{N}$. The PLA Si:N system consists of Si crystals which were im- 
(a) PLA Si:N

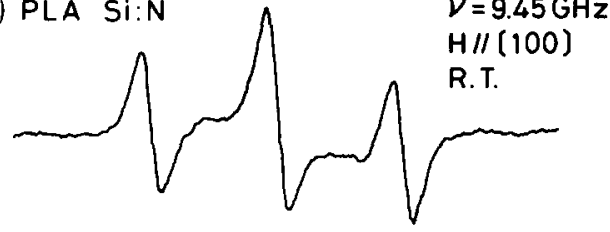

(b) CW-LA Si:N

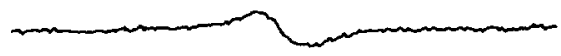

(c) bulk Si:N

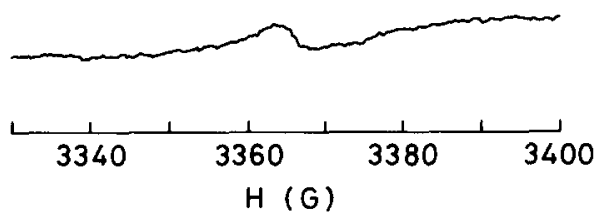

FIG. 1. Typical ESR spectra of the (a) pulsed-laser annealed Si:N $\left(2 \times 10^{14}\right.$ $\left.\mathrm{N}_{2}{ }^{+} / \mathrm{cm}^{2}\right),(\mathrm{b}) \mathrm{cw}$ laser annealed Si:N $\left(5 \times 10^{14} \mathrm{~N}_{2}{ }^{+} / \mathrm{cm}^{2}\right)$, and (c) bulk Si:N doped uniformly with the concentration of $6 \times 10^{15} \mathrm{~N} / \mathrm{cm}^{3}$.

planted with $1 \times 10^{13}-1 \times 10^{16} \mathrm{~N}_{2}^{+} / \mathrm{cm}^{2}$ at $70 \mathrm{keV}$ followed by $Q$-switched ruby laser annealing at $1.3 \mathrm{~J} / \mathrm{cm}^{2}$ in air. This laser energy density yields the high reflectivity phase for a duration of $120 \mathrm{~ns}$ as observed by time-resolved optical reflectivity measurements. ${ }^{5}$ The $\mathrm{cw}$ laser annealed $\mathrm{Si}: \mathrm{N}$ system consists of Si crystals which were implanted with $2 \times 10^{14}$ and $5 \times 10^{14} \mathrm{~N}_{2}^{+} / \mathrm{cm}^{2}$ at $150 \mathrm{keV}$ followed by $\mathrm{cw}$ Ar laser annealing (spot size $56 \mu \mathrm{m}$, scan speed $25 \mathrm{~cm} / \mathrm{s}$, dwelling time $0.22 \mathrm{~ms}$, and scan step $10 \mu \mathrm{m}$ ). The laser powers used were 14 and $10 \mathrm{~W}$ which induced liquid phase regrowth and solid phase annealing, respectively. The bulk Si:N system consists of Si crystals in which $\mathrm{N}$ impurities are uniformly doped with concentrations from $1 \times 10^{15}$ to $6 \times 10^{15} / \mathrm{cm}^{3}$ during Si crystal growth with a special technique. ${ }^{6}$

Typical ESR signals are shown in Fig. 1. They were observed in the three types of samples. In the case of the PLA Si:N, three hyperfine (hf) lines attributed to $N_{s}^{(0)}$ can be seen as shown in Fig. 1(a). On the other hand, no hf lines were observed for both the cw laser annealed Si:N and bulk Si:N, as shown in Figs. $1(\mathrm{~b})$ and $1(\mathrm{c})$. The thermal annealing kinetics of $N_{s}^{(0)}$ centers in PLA Si:N system was estimated to be $\tau^{-1}=(1-100) \times 10^{6} \mathrm{exp}(-1.3 \pm 0.2 \mathrm{eV} / k T) \mathrm{s}^{-1}$, which will be reported elsewhere. This indicates that cw laser annealing and $\mathrm{Si}$ crystal growth maintain high temperatures for long enough times to anneal out $N_{s}^{(0)}$. Therefore, the great effectiveness of PLA for introducing $N_{s}^{(0)}$ in $\mathrm{Si}^{2,3,7}$ is found to be mainly due to both the extremely high velocity $(2-3 \mathrm{~m} / \mathrm{s})$ of recrystallization and the extremely rapid cooling (faster than $\left.10^{9}{ }^{\circ} \mathrm{C} / \mathrm{s}\right)^{5)}$ after recrystallization.

Secondly, ESR measurements were performed on $\mathrm{Si}$ samples which were doubly implanted with $\mathbf{N}$ and $\mathbf{P}$ ions at $70 \mathrm{keV}$, followed by PLA at $1.3 \mathrm{~J} / \mathrm{cm}^{2}$. The average projected ranges of $\mathrm{N}_{2}^{+}$and $\mathrm{P}^{+}$are about 920 and $850 \AA$, respectively. After this double ion implantation, the crystallinity of the implanted Si layer was restored by PLA which activated about $100 \%$ of $\mathrm{P}$ impurities electrically. Both $N_{s}^{(0)}$ and $\mathrm{P}$ are distributed over approximately $2500 \AA$ as a result of PLA; this was determined by ESR measurements after anodic oxidation and etching in hydrofluoric acid.

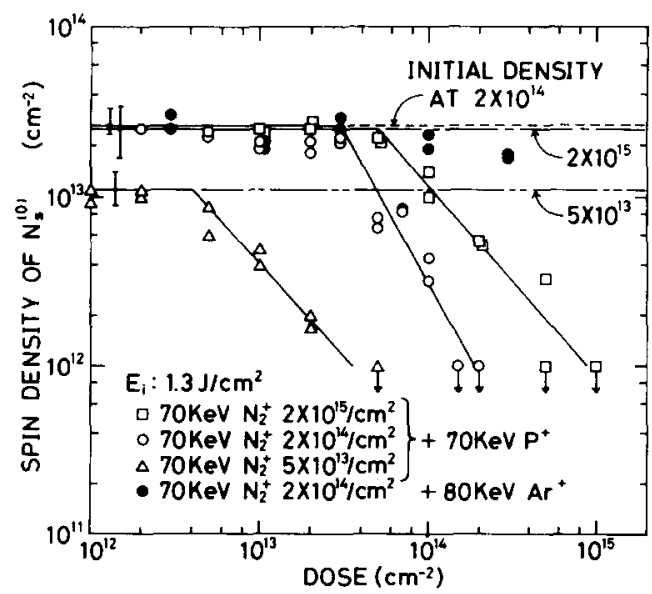

FIG. 2. Effect of phosphorus-donor impurity introduction in PLA Si:N samples which were formed by $5 \times 10^{13}, 2 \times 10^{14}$, and $5 \times 10^{14} \mathrm{~N}_{2}+/ \mathrm{cm}^{2}$ implantation and subsequent PLA, respectively. The density of $N_{s}^{(0)}$ is plotted as a function of $\mathbf{P}^{+}$dose for PLA Si:N:P samples. The result for $\mathrm{Ar}^{+}$ implantation is also shown for comparison, where no significant change in spin density of $N_{s}^{(0)}$ can be seen.

Figure 2 shows the effect of $P$ impurity introduction in the PLA Si:N system. It can be seen that the spin density of $N_{s}^{(0)}$ decreases with $\mathbf{P}^{+}$dose above a critical dose for each PLA Si:N sample. There are three possibilities considered for this decrement; (1) pairing of $N_{s}^{(0)}$ and $\mathbf{P}$ impurities, (2) strong spin-spin interaction between localized spin of $N_{s}^{(0)}$ and degenerate electrons originating from $P$ shallow donors, and (3) formation of diamagnetic $N_{s}^{(-)}$as a result of trapping of $\mathrm{P}$ donor electrons by deep level $N_{s}^{(0)}$ centers. The first possibility can be ruled out because the critical $\mathrm{P}^{+}$dose is independent of the spin density of $N_{s}^{(0)}$ and increases with the implanted $\mathrm{N}_{2}^{+}$dose, i.e. $4 \times 10^{12}, 3 \times 10^{13}$, and $5 \times 10^{13} \mathrm{P}^{+} /$ $\mathrm{cm}^{2}$ for the PLA Si:N samples formed with $5 \times 10^{13}$, $2 \times 10^{14}$, and $5 \times 10^{14} \mathrm{~N}_{2}{ }^{+} / \mathrm{cm}^{2}$, respectively. The second possibility is very interesting, but may also be ruled out because no significant ESR line broadening and $g$ shift due to such an interaction have been observed in iron impurities with $d$ electrons in degenerate $\mathrm{Si}: \mathrm{P}^{8}$ or dangling bonds of $\mathrm{Si}$ in implanted,degenerate $\mathrm{Si}: \mathbf{P}$ systems. ${ }^{9}$

The experimental results shown in Fig. 2 can be interpreted qualitatively by the third possibility as followed. No significant changes in the spin density of $N_{s}^{(0)}$ are seen up to the $\mathrm{P}^{+}$critical doses. This is thought to be due to trapping of $\mathrm{P}$ donor electrons by $\mathrm{N}$-related defects ${ }^{3}$ except $N_{s}$ which are induced by PLA, as illustrated in Figs. 3(a) and 3(b). Because these $\mathrm{N}$-related point defects (designated $\Sigma N$, etc.) increase with $\mathrm{N}_{2}{ }^{+}$dose, ${ }^{3}$ the critical dose increases with $\mathrm{N}_{2}{ }^{+}$dose. The Fermi level rises with the $\mathbf{P}$ donor concentration as illustrated schematically in Figs. 3(a)-3(c). The gradual decre-

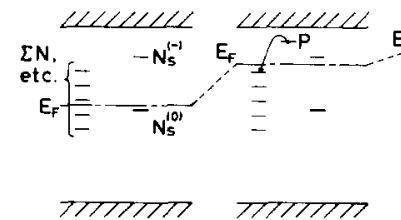

(a)

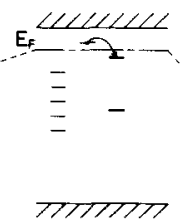

(c)

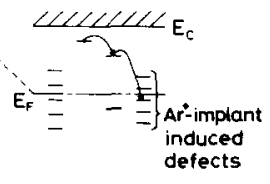

(d)
FIG. 3. Schematic energy-level diagram of impurities and defects in (a) PLA Si:N, (b) PLA Si:N:P with lower concentration of $P$ donors and (c) with higher one, and (d) PLA Si:N:P implanted with a low dose of Ar ion. 


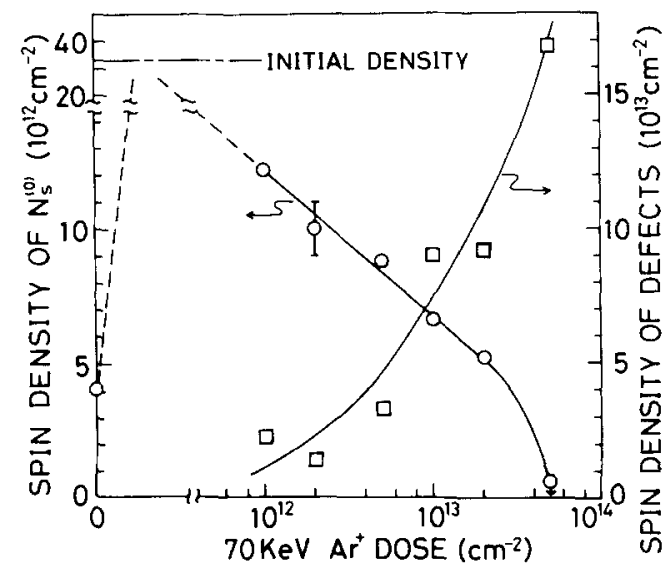

FIG. 4. Effect of Ar ion implantation in PLA Si:N:P system. The densities of $N_{s}^{(0)}$ and implantation-induced point defects are plotted as functions of $\mathrm{Ar}^{+}$dose.

ment of $N_{s}^{(i)}$ with $\mathrm{P}^{+}$dose suggests that the probability that the $N_{s}^{(-1}$ level is occupied at RT increases gradually with $\mathbf{P}$ donor concentration. Consequently, the $N_{s}^{(-)}$level seems to be located near the $\mathrm{P}$ donor level as illustrated in Fig. 3(c).

Lastly, in order to test the third explanation, we investigated the effects point defects have upon the spin density of deep level $N_{s}^{(0)}$ centers. In this case, the point defects were introduced by 70-keV Ar ion implantation in PLA Si:N:P samples. Figure 4 shows a typical result for the Si:N:P system which was formed by double ion implantation with 70$\mathrm{keV}, 2 \times 10^{14} \mathrm{~N}_{2}+/ \mathrm{cm}^{2}$ and $1 \times 10^{14} \mathrm{P}^{+} / \mathrm{cm}^{2}$ and subsequent PLA at $1.3 \mathrm{~J} / \mathrm{cm}^{2}$. It can be seen that $\mathrm{Ar}$ ion implantation with $1 \times 10^{12} / \mathrm{cm}^{2}$ increases the spin density of $N_{s}^{(0)}$ by $8 \times 10^{12} / \mathrm{cm}^{2}$ and the spin density of $N_{s}^{(0)}$ recovers to $38 \%$ of that $\left(3.2 \times 10^{13} N_{s}^{(0)} / \mathrm{cm}^{2}\right)$ of the initial Si:N system in spite of the production of point defects. The increment seen up to a dose of $2 \times 10^{13} / \mathrm{cm}^{2}$ becomes smaller with $\mathrm{Ar}^{+}$dose. This increment is interpreted in terms of trapping of the second electron of $N_{s}^{(-)}$by Ar-implantation induced point defects with deeper levels and lowering of the Fermi level as shown in Fig. 3(d), but is not explained by pairing of $N_{s}^{(0)}$ and $P$ impurities. Above the dose of $2 \times 10^{13} \mathrm{Ar}^{+} / \mathrm{cm}^{2}, N_{s}^{(0)} \mathrm{de}-$ creases with $\mathrm{Ar}^{+}$dose below the initial density $\left(4 \times 10^{12}\right.$
$\left.N_{s}^{(0)} / \mathrm{cm}^{2}\right)$ of the Si:N:P system, as expected from heavy damage of PLA Si:N:P crystal. According to a perturbative calculation by Pantlides ${ }^{4}$, the $N_{s}^{1-1}$ center binds two electrons which have the first ionization energy of $52.5 \mathrm{meV}$ and the second ionization energy of $335.9 \mathrm{meV}$. The former is located near to the level of $P$ donor ( $46 \mathrm{meV}$ ), which is consistent with our interpretation. However, it is obviously necessary to do more investigations to clarify the existence or energy level of $N_{s}{ }^{(-)}$center.

In conclusion, the substitutional nitrogen impurities with neutral charge, $N_{s}^{(0)}$, can be introduced by pulsed-laser annealing of $\mathrm{N}_{2}^{+}$-implanted Si. So far, we have been unable to introduce $N_{s}^{(0)}$ by either cw laser annealing of $\mathrm{N}_{2}^{+}$-implanted Si or by Si crystal growth with a special doping of $\mathbf{N}$ impurities. Our studies suggest that nitrogen is introduced into substitutional sites under conditions involving rapid recrystallization $(2-3 \mathrm{~m} / \mathrm{s})$ and extremely rapid cooling rates $\left(>10^{9}{ }^{\circ} \mathrm{C} / \mathrm{s}\right.$ ). The ESR result for doping of $P$ shallow donors into $\mathrm{Si}: \mathrm{N}$ suggests that the substitutional nitrogen impurities with a negative charge, $N_{s}^{(-)}$, are formed in the pulsed-laser annealed Si:N:P system.

The authors would like to thank Professor H. Suematsu for his kind support in ESR measurements, Dr. T. Nishimura and Dr. Y. Akasaka (LSI Lab.; Mitsubishi Electric Co.) for supplying cw laser annealed Si:N samples, and Dr. H. Harada and Dr. T. Abe (Shin-Etsu Handotai Co.) for supplying bulk $\mathrm{Si}: \mathrm{N}$ samples.

'K. L. Brower, Phys. Rev. Lett. 44, 1627 (1980).

${ }^{2}$ K. L. Brower, Phys. Rev. B 26, 6040 (1982).

${ }^{3}$ K. Murakami, K. Masuda, Y. Aoyagi, and S. Namba, Physica B 116, 564 (1983).

${ }^{4}$ S. T. Pantlides and C. T. Sah, Phys. Rev. B 10, 638 (1974).

${ }^{5}$ K. Murakami, H. Itoh, K. Takita, and K. Masuda, Physica $B 117$ \& 118, 1024 (1983).

${ }^{6} \mathrm{~T}$. Abe, K. Kikuchi, S. Shirai, and S. Muraoka, in Semiconductor Silicon, edited by H. R. Huff, R. J. Krieger, and Y. Takeishi (Electrochemical Society, Pennington, New Jersey, 1981), p. 54.

${ }^{7}$ H. J. Stein, Appl. Phys. Lett. 43, 296 (1983).

${ }^{8}$ T. A. Kennedy and J. H. Pifer, Phys. Rev. B 11, 2017 (1975).

${ }^{9}$ K. Murakami, K. Masuda, K. Gamo, and S. Namba, Appl. Phys. Lett. 30 , 300 (1977). 